\title{
The profile of enzymes relevant to solvent production during direct fermentation of sago starch by Clostridium saccharobutylicum P262 utilizing different pH control strategies
}

\begin{abstract}
The profile of enzymes relevant to solvent production during direct fermentation of sago starch by Clostridium saccharobutylicum P262 in a 2 L stirred tank fermenter was determined utilizing different $\mathrm{pH}$ control strategies. During fermentation without $\mathrm{pH}$ control (initial $\mathrm{pH}$ of 6), the specific activity of crotonase, thiolase, and $\beta$-hydroxybutyryl-CoA dehydrogenase increased proportionally with solvent production. The highest crotonase $(3,450.7 \mathrm{~kat})$ and phosphotransbutyrylase activity $(1,475.6 \mathrm{~kat})$ was observed in fermentation where $\mathrm{pH}$ was maintained at 5 during the acidogenic phase and corresponded to a fairly high acid accumulation but low solvent production. During fermentation with a controlled $\mathrm{pH}$ of 5.25 during the sol-ventogenic phase, the highest thiolase specific activity (255.7 kat) was obtained and corresponded to the highest production of acetone. On the other hand, the highest specific activities of crotonase, $\beta$-hydroxybutyryl-CoA dehydrogenase, and phosphotransbutyrylase were observed at $\mathrm{pH} 5.5$ and corresponded to the highest production of ethanol and butanol. Butyryl-CoA dehydrogenase had no significance role in solvent fermentation. These results suggested that $\mathrm{pH}$ control strategies were important for improvement of solvent production during direct fermentation of sago starch by $\mathrm{C}$. saccharobutylicum.
\end{abstract}

Keyword: sago starch, Clostridium saccharobutylicum, solventogenesis, acetogenesis, enzymes relevant to solvent fermentation 